\title{
Intermediary Multicentric Prospective and Comparative Analysis of 435 Mobile Bearing Total Knee Arthroplasties of Ultra Congruent Stabilization Mechanism Versus Peg and Cam Stabilization Mechanism. A Point-in-Time Analysis of the Orthowave ${ }^{\mathrm{TM}} 6$ Database
}

\author{
Dr JM Durand ${ }^{1}$, Dr P Viale 1 , Pr P Massin ${ }^{2}$, Dr JL Cotte and Dr C Jardin $^{4}$
}

\section{Introduction}

The Rolflex TONIC total knee implant was launched in early 2016. It is a cruciate sacrificing design and offers a choice of 2 cruciate substituting mechanism according to the UC (Ultra-Congruent) concept or to the PS (Postero-stabilisation with peg and cam) concept. The PS choice can be associated to a fixed tibial bearing or to a mobile tibial bearing, while the UC choice can only be associated to a mobile tibial bearing. The international use of UC total knee prosthesis is low: according to the 2019 AJRR report the UC variant was up at $4.5 \%$ of use in 2018 , while the PS variant accounted for the largest frequency of use at 51.6\%. The second most used type of TKA was the cruciate retaining (CR) variant at $43.8 \%$ of use. There is currently no CR variant in the Rolflex TONIC portfolio.

In order to inform of any differences in terms of etiology, indications, patient profile, surgical choices, and clinical and functional performance between the PS and the UC cruciate substituting mechanisms, this document will analyze only the mobile bearing variants of the Roflex TONIC UC and PS. The patients implanted with Fixed bearing PS will not be included in this analysis.

A prospective clinical follow-up of the Rolflex TONIC has been organized by the sponsor (Evolutis, Briennon, France) to evaluate the safety and performance of this new device. This study includes the implants used since June 2016 and up to December 2018. The study design will review the patients at 2, 5 and 10 years of follow-up. At the date of this intermediary report, the 2 years review is not yet terminated. The 2 years report is expected for early 2021 when all patients included will show more than 2 years of FU. Therefore, this intermediary analysis should only be viewed as a security control analysis in search for any anticipated deviation

${ }^{1}$ Hôpital Privé Guillaume de Varye, Saint-Doulchard, France

${ }^{2}$ Clinique Hartmann, Neuilly, France

${ }^{3}$ Polyclinique Sainte-Marguerite, Auxerre, France

${ }^{4}$ Clinique des Ormeaux, Le Havre, France in the expected results. The average length of follow-up will remain short until all patients will be reviewed at 2 years of minimal FU, yet it will evidence if any short or mid-term complication occurred, and how good is the recovery of the patients estimated through an IKS and an OXFORD scores.

\section{Patients}

Between June 2016 and December 2018, the 5 evaluators operating in 4 orthopaedic centers, have recorded 435 total knee prosthesis (412 patients) with a mobile bearing in the Orthowave ${ }^{\mathrm{TM}} 6$ database. The patients were admitted for primary surgery in $99.5 \%$ of the cases, and for revision in $0.5 \%$ ( 4 cases). The mean age of the patients at operation time was 74.5, and when comparing the PS versus the UC group, there is an extremely significant difference for age between the 2 groups: 79.1 for the PS group versus 70 for the UC group (PS Group mean Age 79.12 (38 -> 94), standard deviation 6.45, UC Group mean Age 70.02 (48 -> 89), standard deviation 7.43, Test Student-Fischer ( $t)$ : -13.649, p value: 1.620909e-35 (+++) : $p<0,001$ : extremely significant difference between groups).

The etiology was rather conventional with $95.8 \%$ of arthritis, $1.9 \%$ of necrosis, $0.9 \%$ of revision, $0.7 \%$ of inflammatory arthritis, and $0.7 \%$ of post-trauma sequalae.

The patients were ASA 1 in $10.05 \%$ of the cases, ASA 2 in $56.44 \%$, and ASA 3 in $35.51 \%$. There was no ASA 4 or 5 in the group.

There were $58.3 \%$ of female patients versus $41.7 \%$ of males, with average size of $163.8 \mathrm{~cm}(140-195)$ and weight at $80.6 \mathrm{~kg}$ (41-134), resulting in a BMI at 30.0 (16.4-50.4). 16.3\% of the patients were classified "normal", $37.7 \%$ with a "slight" obesity, $40.7 \%$ with a "medium" obesity, and 5.3\% with a "severe" obesity. And the comparison of the 2 groups show a highly significant difference of BMI between the PS and the UC group: PS Group mean BMI at 29.3 ((20 -> 45.2) standard deviation 5.08) versus UC group mean BMI at 30.77 ((16.4 -> 50.47) standard deviation 5.56). 


\section{Methodology}

The data presented in this document have been extracted from a Monitored Data Base (MDB) hosted in the Orthowave ${ }^{\mathrm{TM}} 6$ database, and analyzed on April 15,2020 . Orthowave ${ }^{\mathrm{TM}} 6$ is dedicated to the recording, protection, and analysis of clinical and functional follow-up data of hip and knee arthroplasty procedures. Any participation of an evaluation center in the database requires the purchasing of a license and the use of dedicated access codes.

The data is recorded by each evaluator on his own Orthowave ${ }^{\mathrm{TM}} 6$ account, and is later transferred to the MBD database which is accessible to the sponsor of the study (Evolutis). The data accessible through the MDB is fully confidential and compliant with all European regulations for medical research. The sponsor has no access to the personal data of the patients, and cannot modify the patient files.

The personal recorded data is limited to the gender, the size and the height of the patient, and his(her) birth date. The information related to the surgery include the date of surgery, the description of the implants used, the duration of surgery, the ASA score of the patient. Complications are recorded at any delay of occurrence from intra-operative to late complication. Revisions are recorded through the modification of the status of the patient in the study. And finally, the patient is physically evaluated through an IKS score, and is asked to answer a PROM (Oxford) score at each of the post-operative evaluation.

The IKS score was developed in 2011 by the Knee Society in order to evaluate the results of the total knee arthroplasties on the basis of objective clinical data and the function of the knee, but also on the expectations and on the satisfaction of the patients. The score ranks on a total of 200 including 100 for the knee score and 100 for the function score. A 200 score indicates a perfect knee.

The Oxford Knee Score is a patient self-completion PRO (Patient Reported Outcomes) containing 12 questions on activities of daily living. The OKS has been developed and validated specifically to assess function and pain after TKR. The Oxford score rank between 12 and 60 . The lower the score, the better are the results of the assessed knee: $12=$ perfect knee, $60=$ fully disabled knee.

Orthowave ${ }^{\mathrm{TM}} 6$ include a statistic calculation modulus that enables to calculate descriptive data of the studied population, make group comparison statistics, and calculate a Kaplan-Meier survival curve.

\section{Implants}

All bearings were mobile. For the Rolflex TONIC knee, the mobile bearing tibial baseplate is identical for PS or for UC use. The femoral condyles and the polyethylene insert are both PS or UC depending on the choice of stabilization mechanism, but in both cases the size of the insert is equal to the size of the condyles.

In this analysis the PS to UC ratio was 51 to $49 \%$. Concerning the fixation mode of both the femoral condyles and the tibial baseplates, both groups have a larger share of cementless fixations (Condyles: $64.4 \%$ for the PS vs $77.5 \%$ for the UC, Tibial baseplate $66.5 \%$ for the PS vs $78.4 \%$ for the UC), but the statistical comparison of the groups show an extremely significant difference between the groups (Condyles: Pearson (khi2): 51.863, p value: 3.203633e11 (+++), Tibial baseplate: Pearson (kbi2), p value: 1.921669e-10 (+++): 48.21, $p<0,001$ : extremely significant difference), meaning that the use if cementless components is significantly more frequent in the UC variant.

A patellar resurfacing was associated in $93.8 \%$ of the cases and not resurfaced in only $6.2 \%$ of the cases.

In mobile bearing Rolflex TONIC total knee arthroplasties, the polyethylene inserts are of the same size as the size of the femoral condyles. This sizing method enables the best congruency possible between the condyles and the insert to the benefit of stability, kinematics and wear. The only possible adaptation is on the selection of the thickness of the insert.

In this analysis, the $10 \mathrm{~mm}$ (minimal thickness) inserts have been used in $56.4 \%$ of the surgeries, the $12.5 \mathrm{~mm}$ in $34.9 \%$, and the $15 \mathrm{~mm}$ in $8.7 \%$. No insert of $18 \mathrm{~mm}$ of thickness has been used. The comparison of insert thickness use between groups is highly significant: in the PS group, $61.6 \%$ of the inserts used are of $10 \mathrm{~mm}, 34.2 \%$ are of $12.5 \mathrm{~mm}$, and only $4.1 \%$ are of $15 \mathrm{~mm}$. In the IC group, the comparative frequencies are respectively $50.5 \%, 35.6 \%$ and $13.4 \%$. The statistical comparison demonstrates a highly significant difference between the PS and the UC (chart and table 1).

\section{Results}

371 patients had been evaluated with an IKS questionnaire at a mean 10.4 months after surgery (1.5 37.4). Of which 125 patients had been evaluated at a minimum of 12 months.

The IKS score for the full group was at a mean 172.2 ((64-200) standard deviation 26.01) including a knee score at 91.3 ((39-100) standard deviation 10.27), and a function score at 80.8 ((0-100) standard deviation 20.02).

For the 125 patients with more than 12 months of Follow-up, the IKS score was at a mean 182.3 (108 -> 200) standard deviation: 20.28 . 


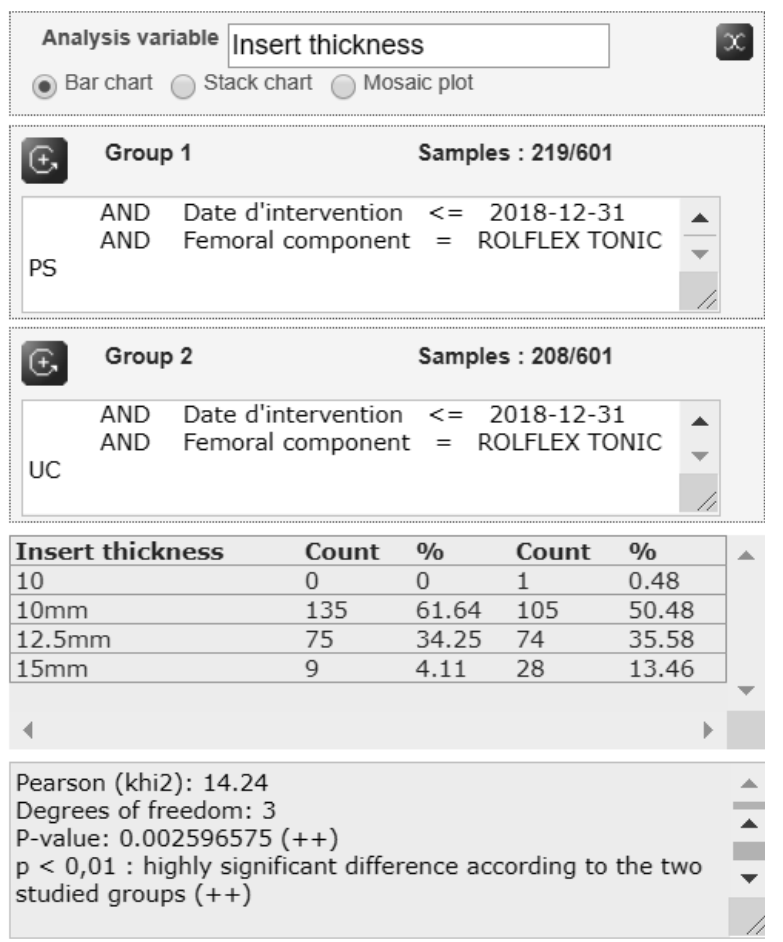

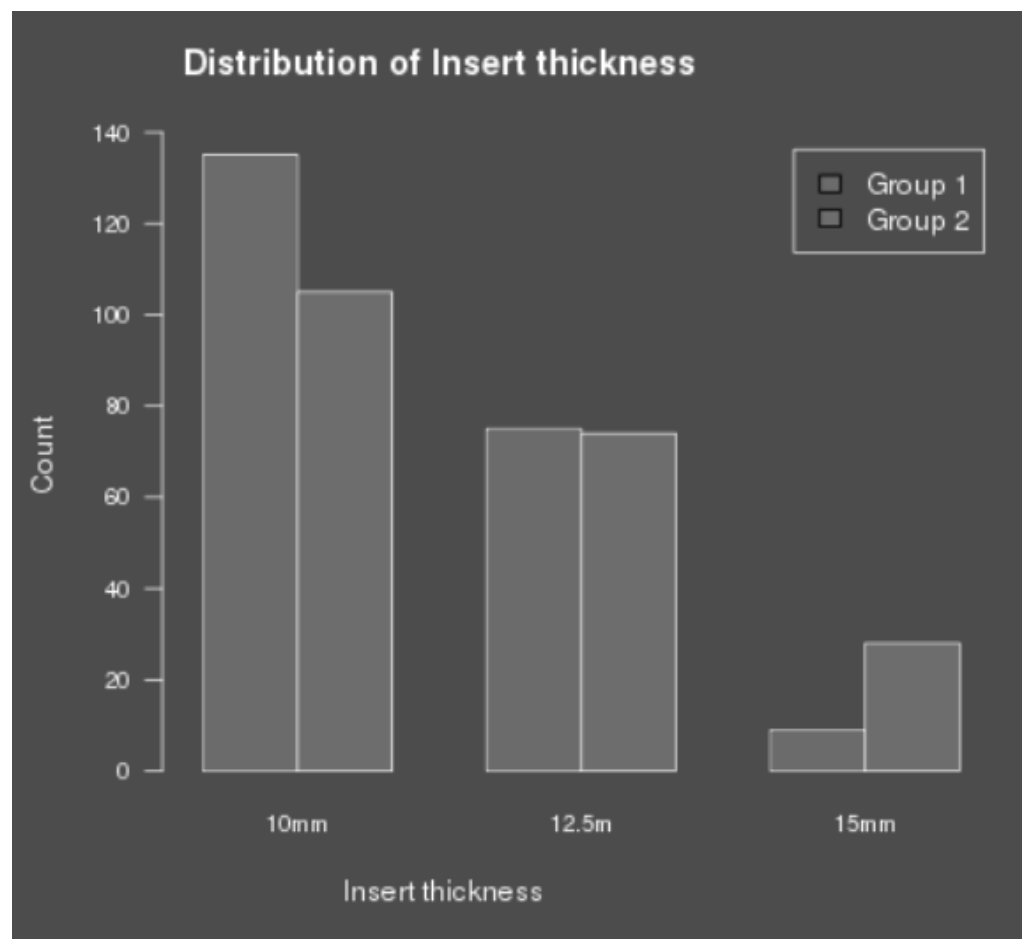

Table 1 and chart: comparative distribution of the thickness of the polyethylene inserts used for the PS versus the UC variants of the Rolflex TONIC

When compared between PS and UC group, the IKS score difference is very highly significant: PS Group at mean value 167.54 (80 -> 200) sd 26.01 versus UC Group at mean value 177.15 (64 -> 200) sd 25.14. The student-Fischer test $(\mathrm{t})$ is calculated 3.615 with a P-value at 0.0003419222 (+++), demonstrating a very highly significant difference according to the two studied groups ( $p<0,001)$, and meaning that the knee and function results for the UC patients are significantly better than for the PS patients.

There were less patients to answer the Oxford questionnaire but at a longer follow-up: 157 patients at 15.5 months of average FU. The mean OXFORD score for the full group is at 18 (12-36) sd 4.47. (Reminder, the Oxford score is rated on a total of 60 , and the lower the score the better the resultsof the assessed knee: $12=$ perfect knee, $60=$ fully disabled knee).

Again, when compared between PS and UC groups, the difference is significant: PS Group mean value at 18.64 (12 -> 36) standard deviation 4.91 versus UC group mean value at 17.13 (12 -> 29) standard deviation 3.66. The Student-Fischer test (t) at -2.116 and with P-value $0.03595311(+)$ demonstrate a significant difference according to the two studied groups $(\mathrm{p}<0,05)$.

Twenty-five (25) complications at each step of the implants use (3 intra-operative, 17 early or 5 latefollow-up) have been recorded. The complete list of complications at each stage of follow-up is listed in table 2 below. This list includes also the cases that have been revised:
The analysis of the status of the patients indicates that 2 patients $(0.5 \%)$ died, and 3 patients $(0.7 \%)$ have been lost to follow-up including 2 that had been evaluated at 17 and 19 months after surgery.

The Lost-to-follow-up patients are patients that are identified and have been contacted, but that refuse to return for evaluation.

There have been 7 revision surgeries. The status of each revision per type of implant is reported in the table 3. The low number of revisions in each group does not allow to conclude to any statistical difference of revision rates between groups.

The reasons for revision are presented in the table 4 below. 2 of the 3 patients lost-to follow-up had previously been evaluated at 17 and 19 months with very good and excellent IKS score. Among the 7 revisions (1.6\%), the documentation indicates that 3 revisions occurred for cause of infection $(0.7 \%)$, and 1 for trauma $(0.2 \%)$ on a defficient osteoporotic patient, leaving 3 revisions $(0.7 \%)$ analyzed as implant related.

The Kaplan Meier survival analysis for Retrieval of all type (status $\mathrm{C} 1+\mathrm{CT}+\mathrm{CF}+\mathrm{B} 3+\mathrm{B} 4+\mathrm{BT}+\mathrm{BF}$ ) shows no difference between the PS and the UC group (Table 5), with a survival estimated at $98.4 \%$ at 3.51 years of maximal FU for the PS group (95\% Confidence Interval: $0.966-1$ ) versus $97.8 \%$ at 3.36 years of maximal FU for the UC group (95\% Confidence Interval: 0.957-1). The calculated P-value at 0.673 (NS) p >0.05 shows a not significant difference. 
List of complications at each stage of follow-up

Table 2.

\begin{tabular}{|c|c|c|c|c|c|c|}
\hline Period & PS or UC & $\begin{array}{c}\text { Delay } \\
\text { (months) }\end{array}$ & Gender & Age & Complication & Revision \\
\hline \multirow[t]{3}{*}{ Intra operative $(0.7 \%)$} & PS & 0 & Male & 82 & Mis-introduction of the intra-femoral rod & No \\
\hline & UC & 0 & Male & 49 & $\begin{array}{l}\text { Tibial crack next to the anterior tuberosity } \\
\text { osteotomy }\end{array}$ & No \\
\hline & PS & 0 & Male & 86 & $\begin{array}{l}\text { Tibial crack occurred during the impaction of } \\
\text { the implant }\end{array}$ & No \\
\hline \multirow{17}{*}{$\begin{array}{l}\text { Early post-operative } \\
(3.9 \%)\end{array}$} & UC & 3 & Female & 67 & Knee stiffness that required a mobilization & No \\
\hline & UC & 4 & Female & 72 & Knee stiffness that required a mobilization & No \\
\hline & UC & 7 & Female & 72 & Fascia-lata syndrome treated by physiotherapy & No \\
\hline & UC & 0.7 & Male & 89 & $\begin{array}{l}\text { Rupture of the quadricipital tendon with } \\
\text { infection requiring lavage, implant retrieval and } \\
\text { repair }\end{array}$ & Yes \\
\hline & PS & 18 & Female & 75 & $\begin{array}{l}\text { Lateral neuropathy pain with fascia-lata } \\
\text { syndrome that healed spontaneously }\end{array}$ & No \\
\hline & UC & 4 & Female & 70 & $\begin{array}{l}\text { Secondary valgus locking of the tibial implant } \\
\text { following lateral plateau collapse at } 4 \text { months } \\
\text { Revised for a cemented implant }\end{array}$ & Yes \\
\hline & UC & 0.7 & Female & 62 & Carential osteoporosis by bypass at 3 weeks & Yes \\
\hline & UC & 9 & Male & 49 & $\begin{array}{l}\text { Metaphyseal tibial fracture after } 9 \text { months } \\
\text { treated with conservative treatment }\end{array}$ & No \\
\hline & PS & 2 & Male & 78 & Implant subsidence (revised) & Yes \\
\hline & PS & 2 & Female & 80 & Hemarthrosis & No \\
\hline & PS & 0.7 & Male & 79 & Infection at streptococus dysgalactiae (revised) & Yes \\
\hline & PS & 3 & Female & 75 & Patellar dislocation: repair of the medial tendon & No \\
\hline & PS & 6 & Female & 71 & Crepitus of a non-resurfaced patella & No \\
\hline & UC & 11 & Female & 69 & Patellar tendinopathy & No \\
\hline & UC & 1 & Female & 64 & $\begin{array}{l}\text { Skin necrosis with exposure of implants } \\
\text { requiring amputation of the thigh }\end{array}$ & No \\
\hline & PS & 3 & Female & 86 & Cicatricial scarring & No \\
\hline & PS & 6 & Female & 79 & $\begin{array}{l}\text { Progressive chondromalacia patellar pain on a } \\
\text { non-resurfaced patella requiring a secondary } \\
\text { patellar replacement }\end{array}$ & No \\
\hline \multirow[t]{5}{*}{$\begin{array}{l}\text { Late post-operative } \\
(1.15 \%)\end{array}$} & UC & 21 & Male & 67 & $\begin{array}{l}\text { Revision of the prosthesis for absence of } \\
\text { fixation of the tibial component }\end{array}$ & Yes \\
\hline & PS & 15 & Female & 82 & Crepitus of a non-resurfaced patella & No \\
\hline & PS & & Female & 89 & Saphenous nerve syndrome & No \\
\hline & PS & 18 & Male & 85 & $\begin{array}{l}\text { Hematogenous staphylococus lugdunensis } \\
\text { infection }\end{array}$ & Yes \\
\hline & PS & 21 & Female & 84 & Patellar crepitus and quadricipital tendinopathy & No \\
\hline
\end{tabular}

The Kaplan Meier survival analysis for Implant related revision $(\mathrm{C} 1+\mathrm{CT})$ also shows no significant difference between the PS and UC groups (Table 6) although no C1, CF or CT status was attached to the PS group. But since only 2 implant related failures were recorded within the UC group, the number is not significant enough to calculate a statistical difference. For the PS group the survival estimate is $100 \%$ at 3.51 years of maximal FU versus 98.9\% at 3.36 years of maximal FU for the UC group (95\%
Confidence Interval: 0.974-1). The calculated P-value at 0.155 (NS) $p>0.05$ shows a not significant difference.

For the full group, the Kaplan Meier analysis for Retrieval of all type (status C1 + CT + CF + B3 + B4 + BT + BF) calculates a survival rate at $98.3 \%$ (95\% Confidence Interval: 0.97-0.995) at 3.54 years of maximal FU (Table 7), while when restricted to the Implant related failures, the survival rate is at $99.5 \%$ (95\% Confidence Interval: 0.988-1) (Table 8). 
Status and frequency of patient per variant of implant

Table 3.

\begin{tabular}{|l|c|c|c|c|}
\hline Status & \multicolumn{2}{|c|}{ PS } & \multicolumn{2}{c|}{ UC } \\
\hline & Number & $\%$ & Number & $\%$ \\
\hline A: on file & 216 & 97.3 & 207 & 97.18 \\
\hline B1: Lost to Follow-up & 1 & 0.45 & 2 & 0.94 \\
\hline B2: Dead & 2 & 0.9 & 0 & 0 \\
\hline B3: Retrieval (infection/not implant related) & 2 & 0.9 & 1 & 0.47 \\
\hline B4: Retrieval (trauma/not implant related) & 0 & 0 & 1 & 0.47 \\
\hline B5: Out of study & 0 & 0 & 0 & 0 \\
\hline BF: Femoral retrieval (not implant related) & 0 & 0 & 0 & 0 \\
\hline BT: Tibial retreival (not implant related) & 1 & 0.45 & 0 & 0 \\
\hline C1: Failure (retrieval) & 0 & 0 & 1 & 0.47 \\
\hline C2: Failure (clinical) & 0 & 0 & 0 & 0 \\
\hline C3: Failure (radiological) & 0 & 0 & 0 & 0 \\
\hline CF: Femoral failure (implant related) & 0 & 0 & 0 & 0 \\
\hline CT: Tibial failure & 0 & 0 & 1 & 0.47 \\
\hline
\end{tabular}

Details of status for each patient excluded of the study

Table 4.

\begin{tabular}{|c|c|c|c|c|c|c|}
\hline $\begin{array}{l}\text { Patient } \\
\text { Number }\end{array}$ & Status & $\begin{array}{l}\text { Surgery } \\
\text { date }\end{array}$ & Femur & Surgeon & Last eval & Comments \\
\hline 000462664445 & Lost to FU & $09 / 06 / 2016$ & $\begin{array}{l}\text { ROLFLEX } \\
\text { TONIC UC }\end{array}$ & JLC & $30 / 01 / 2018$ & $\begin{array}{l}\text { Female Medium obesity } 77 \text { yo Refuses to return IKS } 189 \text { at } 19 \\
\text { mois }\end{array}$ \\
\hline 000462705940 & Lost to FU & $16 / 06 / 2016$ & $\begin{array}{l}\text { ROLFLEX } \\
\text { TONIC UC }\end{array}$ & JLC & $13 / 12 / 2017$ & Female 65 yo Refuses to answer IKS at 200 at 17 months \\
\hline 001131883992 & Lost to FU & $04 / 06 / 2016$ & $\begin{array}{l}\text { ROLFLEX } \\
\text { TONIC PS }\end{array}$ & PV & $04 / 06 / 2016$ & Female Medium obesity 83 yo Never evaluated \\
\hline 001133052915 & Deceased & $26 / 04 / 2018$ & $\begin{array}{l}\text { ROLFLEX } \\
\text { TONIC PS }\end{array}$ & JMD & $10 / 08 / 2018$ & \\
\hline 001133106203 & Deceased & $14 / 12 / 2018$ & $\begin{array}{l}\text { ROLFLEX } \\
\text { TONIC PS }\end{array}$ & PV & $14 / 12 / 2018$ & \\
\hline 001132625441 & $\begin{array}{l}\text { B3 retrieval } \\
\text { (infection/not imp. } \\
\text { related) }\end{array}$ & $10 / 10 / 2016$ & $\begin{array}{l}\text { ROLFLEX } \\
\text { TONIC PS }\end{array}$ & JMD & 01/09/2017 & $\begin{array}{l}\text { Male Mild obesity } 79 \text { yo Arthroscopic lavage at } 1 \text { month Evidence } \\
\text { of streptococus dysgalactiae Negative evolution Removal of } \\
\text { implants }\end{array}$ \\
\hline 001182663780 & $\begin{array}{l}\text { B3 retrieval } \\
\text { (infection/not imp. } \\
\text { related) }\end{array}$ & $18 / 03 / 2017$ & $\begin{array}{l}\text { ROLFLEX } \\
\text { TONIC UC }\end{array}$ & PM & $18 / 03 / 2017$ & $\begin{array}{l}\text { Female Normal weight } 64 \text { yo Skin necrosis with exposure of } \\
\text { implants at } 1 \text { month Thigh amputation }\end{array}$ \\
\hline 001132627337 & $\begin{array}{l}\text { B3 retrieval } \\
\text { (infection/not imp. } \\
\text { related) }\end{array}$ & $22 / 12 / 2016$ & $\begin{array}{l}\text { ROLFLEX } \\
\text { TONIC PS }\end{array}$ & JMD & $19 / 04 / 2019$ & $\begin{array}{l}\text { Male Medium obesity } 85 \text { yo Hematogenous staphylococus } \\
\text { lugdunensis infection at } 18 \text { months }\end{array}$ \\
\hline 001132105519 & $\begin{array}{l}\text { B4 retrieval } \\
\text { (trauma./not imp. } \\
\text { related) }\end{array}$ & $01 / 02 / 2018$ & $\begin{array}{l}\text { ROLFLEX } \\
\text { TONIC UC }\end{array}$ & JMD & $22 / 02 / 2018$ & $\begin{array}{l}\text { Female Medium obesity } 62 \text { yo Carential osteoporosis by bypass } \\
\text { at } 3 \text { weeks }\end{array}$ \\
\hline 001132286867 & $\begin{array}{l}\text { BT tibial retrieval (not } \\
\text { imp. related) }\end{array}$ & $15 / 06 / 2018$ & $\begin{array}{l}\text { ROLFLEX } \\
\text { TONIC PS }\end{array}$ & PV & $31 / 08 / 2018$ & Male Medium obesity 78 yo Subsidence at 2 months \\
\hline 001132126705 & C1 failure (retrieval) & $27 / 11 / 2017$ & $\begin{array}{l}\text { ROLFLEX } \\
\text { TONIC UC }\end{array}$ & JMD & $09 / 08 / 2018$ & $\begin{array}{l}\text { Female Medium obesity } 70 \text { yo Secondary valgus locking of tibial } \\
\text { implant following lateral plateau collapse at } 4 \text { months Revision at } \\
10 \text { months for a cemented Revision implant }\end{array}$ \\
\hline 001132644336 & CT tibial failure & $16 / 01 / 2017$ & $\begin{array}{l}\text { ROLFLEX } \\
\text { TONIC UC }\end{array}$ & JMD & $26 / 05 / 2017$ & $\begin{array}{l}\text { Male Mild obesity } 67 \text { yo Revised at } 21 \text { months for absence of } \\
\text { fixation of the tibial component confirmed in July } 20818\end{array}$ \\
\hline
\end{tabular}




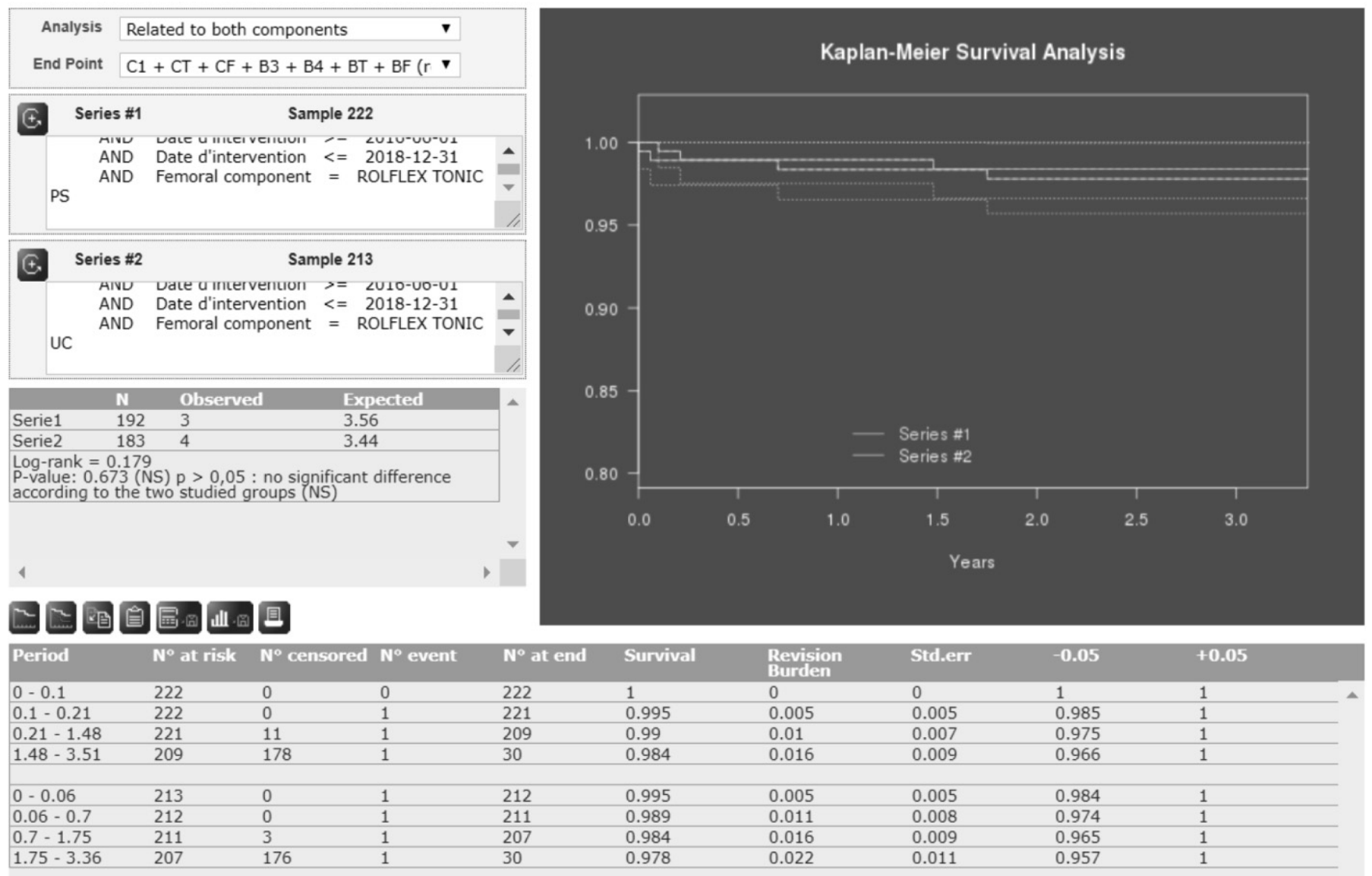

Table 5 and chart: Kaplan-Meier analysis - PS vs UC group - Retrieval of all type $(C 1+C T+C F+B 3+B 4+B T+B F)$

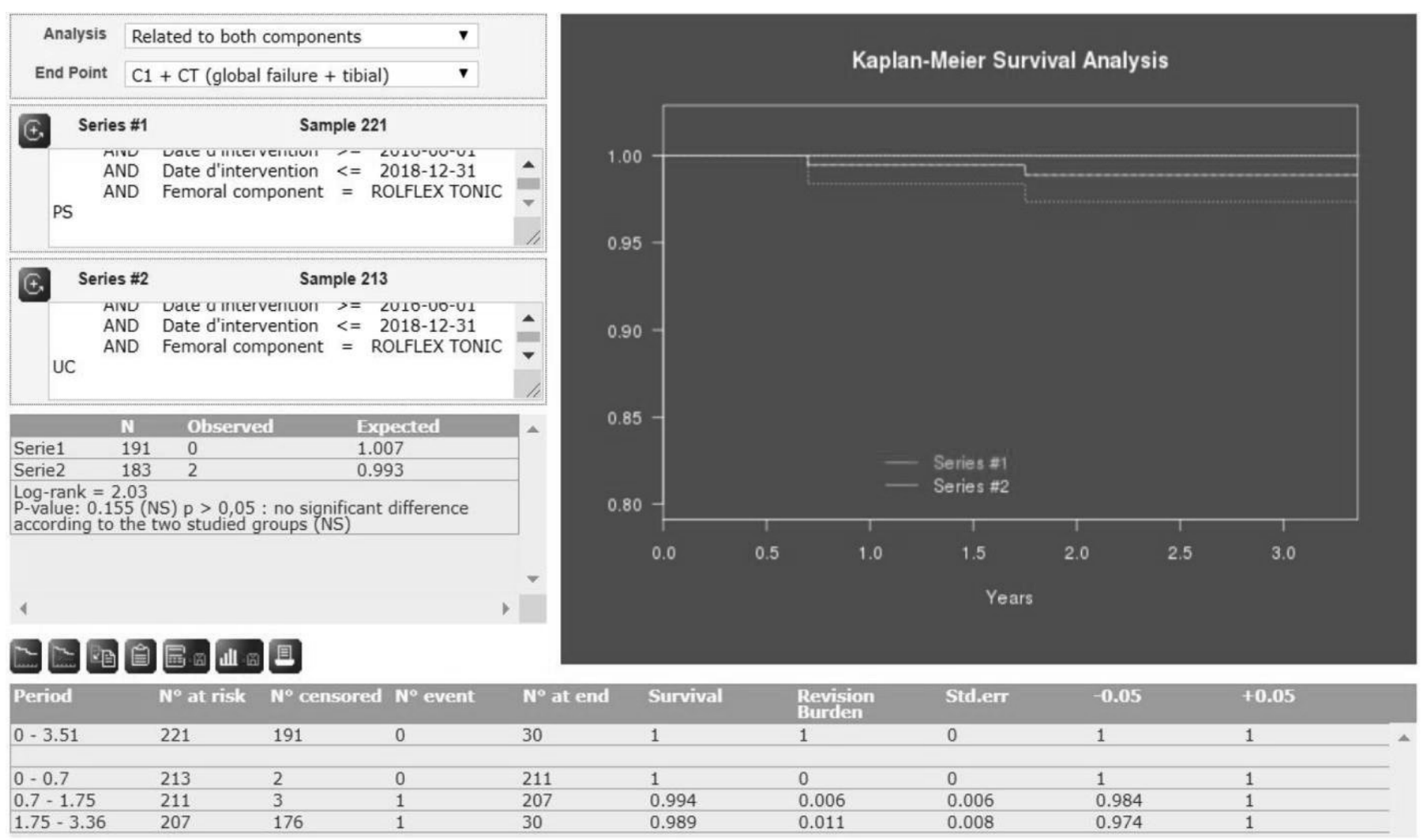

Table 6 and chart: Kaplan-Meier analysis - PS vs UC group - Implant related revision $(C 1+C T)$ 


\section{Kaplan Meier analysis - Full group - Retrieval of all type $(\mathrm{C} 1+\mathrm{CT}+\mathrm{CF}+\mathrm{B} 3+\mathrm{B} 4+\mathrm{BT}+\mathrm{BF})$}

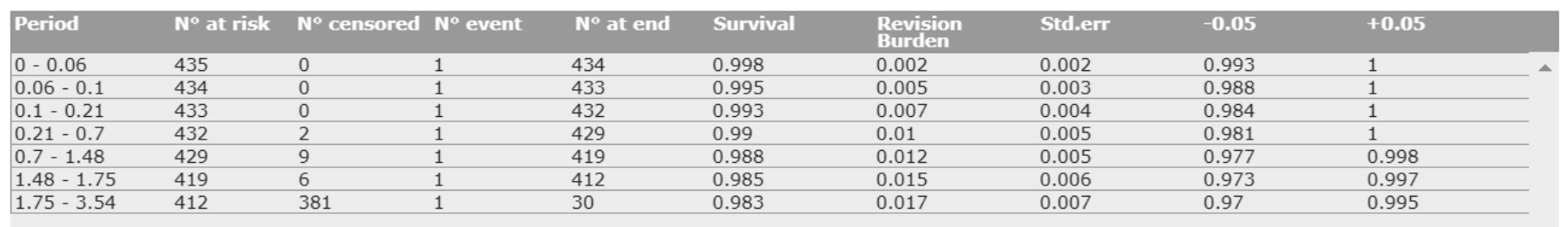

Kaplan Meier analysis - Full group - Implant related revision (C1 + CT)

Table 8.

\begin{tabular}{|c|c|c|c|c|c|c|c|c|c|c|}
\hline Period & $\mathrm{N}^{\circ}$ at risk & $\mathbf{N}^{\circ}$ censored & $N^{\circ}$ event & $\mathbf{N}^{\circ}$ at end & Survival & $\begin{array}{l}\text { Revision } \\
\text { Burden }\end{array}$ & Std.err & -0.05 & +0.05 & \\
\hline $0-0.7$ & 434 & 5 & 0 & 429 & 1 & 0 & 0 & 1 & 1 & $\Delta$ \\
\hline $0.7-1.75$ & 429 & 16 & 1 & 412 & 0.997 & 0.003 & 0.003 & 0.993 & 1 & \\
\hline $1.75-3.54$ & 412 & 381 & 1 & 30 & 0.995 & 0.005 & 0.004 & 0.988 & 1 & \\
\hline
\end{tabular}

\section{Discussion}

Despite the short follow-up associated to the analyzed dataset, and the fact that the collection of data is still under way, the comparison of the complications, clinical results, and survival of the Rolflex TONIC PS with the Rolflex TONIC UC is made possible thanks to the high number of patients included by the 5 surgeon-evaluators since June 2016. The analysis is based on 219 PS implants versus 209 UC implants with an average follow-up of 10.5 months.

The data analysis demonstrates that there are some significant differences in the casuistic of the 2 groups: in comparison to the UC patients, the PS patients are: - Older by 9 years on average: 79.1 for the PS group versus 70 for the UC group - Have a lower average BMI: 29.3 for the PS group versus 30.77 for the UC group.

The age difference is not a surprise as the UC mechanism is best adapted to healthier knees and efficient quadricipital muscle moment. The UC design is also less constrained and requires a good ligamentary status of the knee where the PS more constrained design provides more security in deviated knees. Some of the participating surgeons do not adapt the stabilization design to the profile of each patient, but others, accounting for $80.9 \%$ of the full group, select the stabilization mechanism of the implant according to the patient's profile, including some isokinetic pre-operative measures: for Dr Jean-Marc Durand, a patient with a quadricipital moment of less than $1.6 \mathrm{~kg} / \mathrm{N}$ before surgery is best indicated for a PS variant of the Rolflex TONIC knee.

The BMI difference is more surprising and is not explained by the available dataset. For the average adult population, a BMI less than 25 indicates a "normal weight". When the BMI is above 25 and less than 30 the concerned population is "overweight", and above 30 , the population is "obese". Therefore, this highly significant difference will need to be taken into account when comparing the clinical and survival results of the 2 groups as an increased BMI can potentially reduce the outcomes and increase the complications.

The statistical comparison of patellar resurfacing is not possible due to the low number of resurfaced patellae: $6.2 \%$ within the PS group and 6.4\% within the UC group (Table 9).

But the comparative analysis provided an unexpected information: the proportion of polyethylene insert thicknesses turned out to be highly significantly different between the PS group and the UC group. In the PS group the large majority of the cases (61.6\%) use a $10 \mathrm{~mm}$ thick insert: the minimal thickness available, and only $4.1 \%$ are associated with a $15 \mathrm{~mm}$ thick insert: the highest one available. The PS prosthesis is more constrained than the UC prosthesis, and due to the higher "jump distance" required to subluxate the prosthetic joint (minimum of $14 \mathrm{~mm}$ included in the

Frequency of patellar replacement per variant of Rolflex TONIC

Table 9.

\begin{tabular}{|l|c|l|c|l|}
\hline Patellar Replacement & PS Group & $\%$ & UC Group & $\%$ \\
\hline None & 196 & $93.8 \%$ & 190 & $93.6 \%$ \\
\hline PE implant & 13 & $6.2 \%$ & 13 & $6.4 \%$ \\
\hline
\end{tabular}


design), the PS implant can be used more securely in some deviated primary knees and in some low stage revisions: within the 4 Rolflex TONIC used to treat revision cases in this analysis, only one was a UC type, and was associated with a $15 \mathrm{~mm}$ insert. The 3 others were of PS type with one $10 \mathrm{~mm}$ insert and two $12.5 \mathrm{~mm}$ inserts.

In comparison the UC group used only $51 \%$ of $10 \mathrm{~mm}$ thick inserts, $35.6 \%$ of $12.5 \mathrm{~mm}$ inserts, and as many as $13.4 \%$ (28 cases) of $15 \mathrm{~mm}$ thick inserts.

The available dataset does not provide any information to explain this statistical difference, especially considering that both types of implant are implanted with the exact same instrumentation set. However, it will be highly interesting in the longer follow-up analysis to examine the possible correlation between the increased use of thicker inserts for the UC design, and clinical, functional or survival outcomes.

Both IKS and Oxford score comparison indicate that the clinical and functional outcomes of the UC group are better than the outcomes of the PS group: IKS and Oxford of the PS Group at 167.54 and 18.64 versus IKS and Oxford of the UC Group at 177.15 and 17.13 , both differences are statistically relevant. This difference can be justified by the age difference between the 2 groups with a significant difference of 9 years on average, but can be contradicted by the BMI difference between the 2 groups with a 1.47 point of increased BMI for the UC group. The difference cannot be explained by a different average follow up: the average follow-up for the PS group is 10.4 months for the IKS and 15.9 for the Oxford, versus for the UC group respectively 10.4 and 15.0 .

The Kaplan Meier survival analysis have shown no statistical difference between the 2 groups, would it be for any cause of revision or for implant-related reasons. For any cause of revision, the PS group survival rate is $98.4 \%$ at 3.51 years of maximal FU versus $97.8 \%$ at 3.36 years for the UC group. The statistical difference is not significant. And when the analysis is restricted to the "implant-related" causes alone, the estimate of the PS group is $100 \%$ at 3.51 years of maximal FU versus $98.9 \%$ at 3.36 years of maximal $\mathrm{FU}$ for the UC group. In the analysis also the statistical difference is not significant.

\section{Conclusion}

The current analysis document is for regulatory information purpose only. It has to be considered only as a snapshot of the clinical status of the Rolfex TONIC mobile bearing patients at mid-way of a 2 years review of a multicentric study. The complete results will be available once all patients included in the study up to December 2018 will have been evaluated at 24 months of minimal follow-up, which is expected in semester 1 2021. This document is intended for demonstration of the on-going study, and provides a partial view of the clinical and functional results and an analysis of the complications that have been recorded during the short use of a large number of surgeries. Under this aspect it already provides useful information regarding the safety of use of the device.

The analysis demonstrates very good clinical and functional performance of both variants of the Mobile bearing device. At closely identical length of follow-up the UC variant shows better IKS and Oxford results, but the results of the PS variant are also very good, especially considering that the average age of the patients in the PS group is nearly 10 years older than the average age of the patients in the UC group. And in both groups, the frequency and type of complications, and the survival analysis with a KaplanMeier methodology show identically good results with $0.5 \%$ of implant-related revision for the full group at the maximal follow-up of 3.51 years (mean FU at 10.4 months).

Logically this intermediary analysis will need to be confirmed by the full 2 years review planned for availability in 2021. The full 2 years review will also be an opportunity to evaluate the outcomes related to 2 specific differences that have been identified between the PS and the UC group: the comparative thickness of the polyethylene liners used which showed a tendency for thicker inserts in the UC group, and the average BMI of the patients in each group which showed that the patients selected for a UC variant of the Rolflex TONIC knee are more obese on average than the patients in the PS group. Both of these statistically significant differences will have to be assessed specifically for correlation on the outcomes of the device. 